\title{
Cirurgia minimamente invasiva em chevron e osteotomia percutânea de Bosch no tratamento de hálux valgo. Resultados em médio prazo. Estudo radiológico comparativo*
}

\section{Minimally Invasive Chevron Surgery and Bosch Percutaneous Osteotomy in Hallux Valgus Treatment. Midterm Results. Comparative Radiological Study}

\author{
Juan Manuel Yañez Arauz ${ }^{1,20}$ Nicolás Raimondi ${ }^{1}$ Andrés Eksarho ${ }^{1}$ Diego Lauritto ${ }^{1}$ \\ Maria Eugenia Yañez Arauz ${ }^{1}$ Juan Martin Yañez Arauz ${ }^{2}$ \\ 1 Serviço de Pé e Tornozelo, Hospital Universitario Austral, Buenos \\ Aires, Argentina \\ ${ }^{2}$ Faculdade de Medicina, Universidad Austral, Buenos Aires, Argentina \\ Endereço para correspondência Juan Manuel Yañez Arauz, MD, Gral. \\ Urquiza 340. Acassuso. Buenos Aires, Argentina. PC:1641 \\ (e-mail: juanyanezarauz@gmail.com).
}

Rev Bras Ortop 2022;57(2):250-256.

\author{
Resumo \\ Palavras-chave \\ - procedimentos \\ cirúrgicos \\ minimamente \\ invasivos \\ - hálux valgo \\ - osteotomia \\ - hallux valgus/ \\ diagnóstico por \\ imagem \\ - hallux valgus/cirurgia
}

Objetivo O objetivo deste estudo é comparar os resultados radiológicos da correção angular e sua manutenção no médio prazo entre duas técnicas minimamente invasivas para o tratamento de hálux valgo (cirurgia minimamente invasiva em chevron vs. técnica de Bosch).

Métodos Foi realizada uma análise prospectiva comparativa de pacientes submetidos à cirurgia para deformidade sintomática de hálux valgo. Comparamos duas técnicas minimamente invasivas em grupos homogêneos de população. Dois grupos de 62 e 63 pés, respectivamente, foram constituídos. Comparamos correções angulares de primeiro raio e consolidação, bem como o poder de correção tanto das osteotomias quanto de sua manutenção ao longo do tempo. As complicações pós-operatórias e o tempo cirúrgico em ambos os grupos de estudo também foram avaliados. O seguimento mínimo foi de 2 anos. Resultados Houve diferenças entre ambos os grupos no ângulo intermetatarsal aos 24 meses após a cirurgia. Não houve diferenças entre ambos os grupos em relação ao ângulo metatarsofalângico e ao ângulo articular metatarso-distal. Não houve complicações intraoperatórias em nenhum dos grupos. O tempo cirúrgico entre ambos os grupos apresentou diferenças estatisticamente significativas.

\footnotetext{
Estudo desenvolvido no Hospital Universitario Austral, Buenos Aires, Argentina.
}

recebido

20 de Agosto de 2020

aceito

01 de Dezembro de 2020

Publicado on-line

Novembro 11, 2021
DOI https://doi.org/ 10.1055/s-0041-1729590. ISSN 0102-3616.

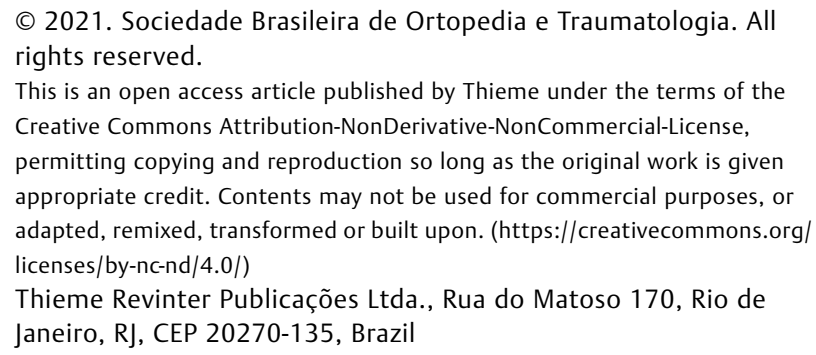

(c) 2021. Sociedade Brasileira de Ortopedia e Traumatologia. All rights reserved.

This is an open access article published by Thieme under the terms of the Creative Commons Attribution-NonDerivative-NonCommercial-License, permitting copying and reproduction so long as the original work is given appropriate credit. Contents may not be used for commercial purposes, or adapted, remixed, transformed or built upon. (https://creativecommons.org/ licenses/by-nc-nd/4.0/) Thieme Revinter Publicações Ltda., Rua do Matoso 170, Rio de Janeiro, RJ, CEP 20270-135, Brazil 


\section{Abstract \\ Keywords \\ - minimally invasive surgical procedures \\ - hallux valgus \\ - osteotomy \\ - hallux valgus/ diagnostic imaging \\ - hallux valgus/ surgery}

Conclusões Sendo as duas técnicas estabilizadas por parafusos, tanto a osteotomia de Bosch quanto a cirurgia minimamente invasiva em chevron (híbrida quando associada à osteotomia percutânea de Akin) apresentam correção adequada de hálux valgo moderado. No entanto, os pacientes tratados com a osteotomia percutânea Bosch apresentaram maior poder de correção do ângulo intermetatarsal no médio prazo, bem como e menor tempo cirúrgico, em relação aos que foram tratados com osteotomia em chevron. Ambas as técnicas apresentaram evolução semelhante ao longo do tempo no que se refere à perda de correção e complicações pós-operatórias.

Objective The purpose of the present study is to compare the radiological results of angular correction and its maintenance in the medium term between two minimally invasive techniques for the treatment of hallux valgus (minimally invasive chevron surgery vs. Bosch technique).

Methods A comparative prospective analysis of patients undergoing surgery for symptomatic hallux valgus deformity was performed. We compared two minimally invasive techniques in homogeneous groups of population. Two groups of 62 and 63 feet respectively, were constituted. We compared first ray angular corrections and consolidation as well as the correction power of both osteotomies and their maintenance over time. The postoperative complications and surgical time in both study groups were also evaluated. The minimum follow-up was 2 years.

Results There were differences between both groups in the intermetatarsal angle at 24 months postsurgery. There were no differences between both groups regarding metatarsophalangeal angle, and distal metatarsal articular angle. There were no intraoperative complications in either group. The surgical time between both groups had statistically significant differences.

Conclusions Both screw-stabilized, Bosch surgery and minimally invasive chevron (hybrid when associated with percutaneous Akin osteotomy) present adequate correction of moderate hallux valgus. However, patients treated with Bosch percutaneous surgery had a greater correction power of the intermetatarsal angle in the medium term, as well as a shorter surgical time, when compared with those who were treated with chevron osteotomy. Both techniques had a similar evolution over time regarding loss of correction and postoperative complications.

\section{Introdução}

O hálux valgo é uma deformidade frequente, complexa e progressiva do antepé, que produz múltiplas manifestações clínicas e tem uma etiologia multifatorial. ${ }^{1}$ A deformidade e as manifestações clínicas dessa patologia ocorrem entre a $3^{a} \mathrm{e}$ a $5^{\underline{a}}$ décadas de vida em $65 \%$ da população. É mais frequente em mulheres. 2,3

Várias técnicas cirúrgicas foram descritas para o tratamento de hálux valgo e a maioria delas inclui uma primeira osteotomia metatarsal. Esta osteotomia pode ser realizada em diferentes áreas do primeiro metatarso: (distal, diafísica média e/ou proximal), associada ou não com liberação de tecido mole. ${ }^{4,5}$

A osteotomia em chevron distal, popularizada por Austin e Leventen, ${ }^{6}$ é aceita como uma boa opção para o tratamento de hálux valgos de leves a moderados, obtendo bons resultados em correção angular e sintomática. ${ }^{7}$
Nas últimas décadas, técnicas percutâneas para o tratamento de hálux valgos foram popularizadas, apoiadas pela teoria do menor tempo cirúrgico, menos trauma cirúrgico, menos dor pós-operatória e recuperação mais rápida. ${ }^{8}$ Peter Bosch modificou a popular osteotomia Kramer, fazendo-a através de uma incisão mínima. Ele usou um fio $\mathrm{K}$ para estabilizar a osteotomia. Em 1990, Bosch ${ }^{9}$ publicou sua técnica de osteotomia percutânea na qual descreveu um deslocamento cefálico lateral de 3/4 do diâmetro mínimo do metatarso, obtendo uma consolidação de $100 \%$. As técnicas percutâneas foram descritas em várias publicações.

Resultados semelhantes foram demonstrados e foram, às vezes, superiores às técnicas abertas. ${ }^{10}$ Contudo, apesar dos resultados desses trabalhos, o tempo de seguimento e os níveis de evidência (III e IV), da cirurgia minimamente invasiva ainda são fonte de debate e controvérsia. ${ }^{11}$

Alguns autores ${ }^{12}$ descreveram procedimentos combinados, utilizando técnicas com incisões mínimas associadas a 
252 Cirurgia minimamente invasiva em chevron e osteotomia percutânea de Bosch Arauz et al.

técnicas puramente percutâneas para tratar uma deformidade do pé. Eles as chamaram de "técnicas híbridas".

O principal objetivo deste estudo é comparar os resultados radiológicos da correção angular e sua manutenção no médio prazo, entre duas técnicas minimamente invasivas para o tratamento de hálux valgos (cirurgia minimamente invasiva em chevron vs. técnica de Bosch).

O objetivo secundário é avaliar as complicações pósoperatórias e o tempo cirúrgico em ambos os grupos de estudo.

A hipótese foi de que a técnica percutânea da Bosch apresenta resultados semelhantes quanto à correção da deformidade e sua manutenção a longo prazo, com menores taxas de complicações e tempo cirúrgico, em comparação com a osteotomia minimamente invasiva em chevron.

\section{Materiais e Métodos}

Foi realizada uma análise prospectiva comparativa dos pacientes submetidos à cirurgia para deformidade sintomática do hálux valgo. Comparamos duas técnicas minimamente invasivas em grupos homogêneos da população quanto à sua deformidade entre primeiro raio, faixa etária e demanda funcional.

De janeiro de 2014 a janeiro de 2015, foram incluídos 125 pés de 96 pacientes, com acompanhamento mínimo de 2 anos. Os principais motivos para a cirurgia foram dor e dificuldade em usar sapatos fechados.

A gravidade da deformidade segundo o ângulo metatarsof -alângico (AMF) pré-operatório foi definida como normal ( $\leq 15$ graus), leve (16-20 graus), moderada (21-39 graus) ou grave ( $\geq 40$ graus). ${ }^{13}$ A presença de um AMF superior a 20 graus no pós-operatório foi definida como deformidade de recorrência. ${ }^{14}$ Apenas pacientes com deformidades moderadas foram incluídos neste estudo.

\section{População}

Dois grupos de pacientes foram formados de acordo com a técnica cirúrgica:

Grupo 1: 62 pés de 48 pacientes. Esse grupo incluiu 4 homens (3 deformidades bilaterais) e 44 mulheres (11 deformidades bilaterais). Todos os pés tinham hálux valgo moderado. Os pés operados incluíam 28 pés esquerdos e 34 pés direitos. A técnica cirúrgica foi uma osteotomia percutânea de Bosch, estabilizada com osteossíntese com parafuso canulado, além do fio K endomedular. Em todos os casos, a liberação do tendão lateral foi realizada por meio de incisão percutânea.

Grupo 2: 63 pés de 48 pacientes. Esse grupo incluiu 3 homens (uma deformidade bilateral) e 45 mulheres (14 deformidades bilaterais). Todos os pés tinham hálux valgo moderado. Os pés operados incluíam 33 pés direitos e 30 pés esquerdos. A técnica cirúrgica foi uma osteotomia distal minimamente invasiva em chevron, estabilizada com parafuso canulado. A - Tabela 1 descreve os grupos.

Todas as cirurgias foram realizadas no mesmo hospital por 3 especialistas em cirurgia no pé e tornozelo. Embora a escolha da técnica não tenha sido aleatória, o cirurgião
Tabela 1 Característica de ambos os grupos

\begin{tabular}{|l|l|l|}
\hline Técnica & Chevron CMI & Bosch \\
\hline Pacientes & 48 & 48 \\
\hline Idade média & 47 & 51 \\
\hline Sexo & $\begin{array}{l}45 \text { mulheres } \\
3 \text { homens }\end{array}$ & $\begin{array}{l}44 \text { mulheres } \\
4 \text { homens }\end{array}$ \\
\hline Lado & 30 esquerda & $\begin{array}{l}28 \text { esquerda } \\
34 \text { à direita }\end{array}$ \\
\hline AMF & 33 à direita & 62 moderados \\
\hline Bilateralidade & 63 moderados & 14 \\
\hline Número de pés & 15 & 62 \\
\hline
\end{tabular}

Abreviaturas: $\mathrm{CMI}$, cirurgia minimamente invasiva; $\mathrm{AMF}$, ângulo metatarsofalângico.

"um" sempre realizou a técnica Bosch para seus pacientes com hálux valgo moderado. Os outros dois cirurgiões realizaram a técnica em chevron minimamente invasiva.

Critérios de inclusão:

1. Hálux valgo moderado sintomático

2. Pacientes com mais de 18 anos.

Critérios de exclusão:

1. Cirurgias anteriores de valgo.

2. Artrite reumatoide ou outra artrite destrutiva.

3. Deformidades graves.

4. Evidência de instabilidade cuneometatarsal.

5. Pacientes com doenças neuromusculares.

\section{Avaliação radiográfica.}

Em ambos os grupos, foram realizadas radiografias em posição de rolamento de peso, em duas incidências: anteroposterior e visão lateral de raios-X. Foram analisados o raio-X no período pré-operatório, aos 3 meses de pós-operatório, e no período pós-operatório distante, após 24 meses. As correções angulares foram comparadas no curto prazo, e a manutenção ou não dessas correções, ao longo de mais de 24 meses, que foi considerado como um pós-operatório distante.

Todas as imagens eram digitais e foram medidas com o software MB Ruler versão 4.0 (MB Software Solutions, LLC, Owings Mills, MD, EUA).

Os autores mediram os seguintes ângulos:

A) ngulo metatarsofalângico (AMF), medido entre o eixo da primeira falange e o primeiro metatarso;

B) ngulo intermetatarsal (AIM), medido pela técnica de medição Miller; ${ }^{15}$

C) Ângulo articular metatarsodistal (AAMD), medido como o ângulo entre o eixo longitudinal do primeiro metatarso e perpendicular à linha desenhada entre a extensão mais medial e lateral da superfície articular metatarsal. ${ }^{16}$

Status e tipo de consolidação da osteotomia foram considerados segundo Jones et al. ${ }^{17}$ A consolidação viciosa foi considerada nos casos em que, nas projeções laterais, a osteotomia metatarsal consolidou-se com um desvio 
angular, tomando como referência o eixo longitudinal do primeiro metatarso. ${ }^{18}$

\section{Análise Estatística}

Os dados obtidos foram incluídos em uma planilha do Excel (Microsoft Corp., Remond, WA, EUA). A perda ou não da correção angular no pós-operatório imediato foi analisada em ambos os grupos de estudo.

Os autores mediram os ângulos das radiografias préoperatórias, bem como as corrigidas, aos 3 e 24 meses de pós-operatório.

Em cada grupo foram registrados os ângulos médios e os desvios-padrão das amostras.

O teste do qui-quadrado de Pearson foi utilizado para a análise de diferenças na distribuição de uma variável em ambos os grupos, considerando um valor de $p<0,05$ estatisticamente significativo.

Para minimizar qualquer efeito possível de uma diferença estatisticamente significativa na medição dos ângulos de ambos os grupos, utilizou-se o percentual de variação dos ângulos, expresso pelas taxas de recuperação (TRs) angular, que é definida pela seguinte equação: diferença entre ângulo pré-operatório e pós-operatório, dividido pelo ângulo préoperatório multiplicado por $100 .{ }^{19}$

Em cada grupo independente, os ângulos no período préoperatório e aos 3 meses de pós-operatório foram comparados usando o teste de Mann-Whitney. Posteriormente, as evoluções de cada ângulo foram comparadas no mesmo grupo aos 3 e 24 meses de pós-operatório, novamente analisadas pelo teste de Mann-Whitney.

Para medir mudanças significativas no mesmo ângulo entre os ângulos pós-operatórios de 3 e 24 meses, utilizamos $o$ teste McNemar.

Para avaliar se houve diferenças na evolução dos três ângulos (AIM, AMF e AAMD) entre ambos os grupos de estudo (técnica Bosch vs. técnica minimamente invasiva em Chevron), utilizou-se o teste $t$ de Student.

Como duas técnicas foram realizadas para o tratamento da mesma patologia, em populações com características semelhantes e homogêneas, buscamos minimizar as influências de variáveis descontroladas.

\section{Resumo da técnica de Bosch}

O paciente é colocado em posição supina, com os pés na borda da mesa cirúrgica, ou ligeiramente fora dela. Isso nos permite posicionar-nos confortavelmente na frente do pé a ser operado, manusear os instrumentos enquanto trabalhamos e usarmos a fluoroscopia.

a) Liberação do tendão da adutora (com técnica percutânea) (-Fig. 1).

b) Incisão paraungueal medial do primeiro dedo, e progressão de um fio K retrógrado de $2,0 \mathrm{~mm}$ para a metáfise distal do primeiro metatarso.

c) A osteotomia metatarsal distal transversal é feita usando uma rebarba (Shannon longa 44), através de um portal medial do pescoço metatarsal, com um corte perpendicular ao eixo do primeiro osso metatarsal. Pos-

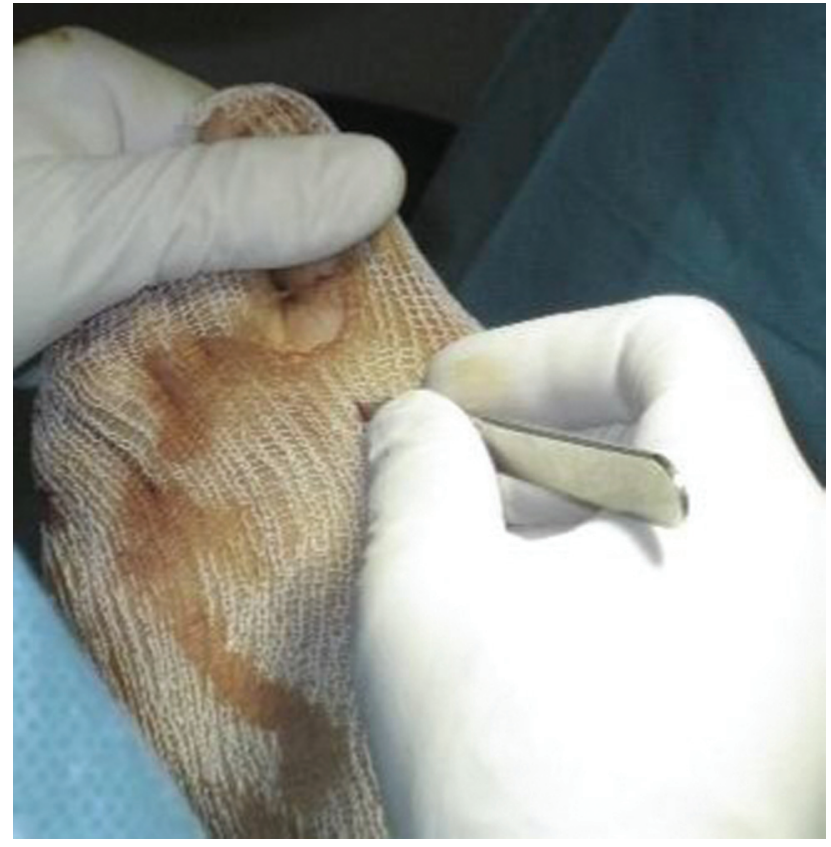

Fig. 1 Liberação percutânea do tendão da adutora.

teriormente, a cabeça metatarsal pode ser deslocada mais de $75 \%$ do diâmetro do primeiro metatarso, se necessário, permitindo correções de AIM graves. É uma osteotomia completa, e deve ser estabilizada com fio $\mathrm{K}$ intramedular. Controle a posição com fluoroscópio em vistas laterais e anteroposterioras.

d) Estabilização utilizando um parafuso canulado ( - Fig. 2).

\section{Resumo da técnica minimamente invasiva em chevron.}

a) O paciente é colocado em posição supina, com os pés na borda da mesa cirúrgica ou ligeiramente fora dela.

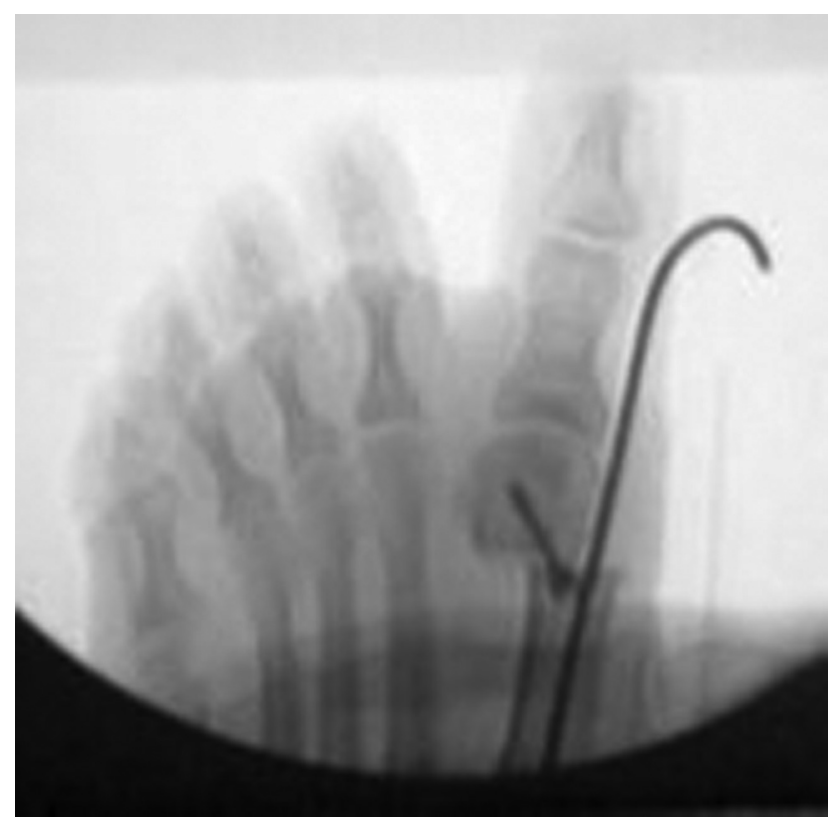

Fig. 2 Técnica Bosch. 
b) Abordagem medial de $30 \mathrm{~mm}$ na primeira cabeça metatarsal medial.

c) "V" ou capsulotomia longitudinal.

d) Bunionectomia da cabeça metatarsal medial.

e) Liberação lateral pela mesma abordagem.

f) Osteotomia em "L" invertido (chevron modificado por Johnson). Deslocamento lateral. Osteossíntese usando um parafuso canulado.

g) Capsuloplastia e síntese de pele.

A gestão pós-operatória e o retorno às atividades foram semelhantes em ambos os grupos. A única diferença foi que na técnica de Bosch, o fio $\mathrm{K}$ foi removido em 3 semanas, e a mobilidade articular ativa e passiva começou a partir daí. Na técnica em chevron, a mobilidade conjunta começou em 2 semanas de pós-operatório.

\section{Resultados}

A idade média dos pacientes foi de 49,3 anos (faixa, 77-19). Sete pacientes eram homens e 89 mulheres.

Foram incluídos 58 pés esquerdos e 67 direitos. Vinte e nove pacientes apresentaram patologia cirúrgica bilateral.

No grupo 1 (Bosch), a correção média para o AMF, AIM e AAMD foi de $20,19^{\circ}$; $8,66^{\circ}$; e $9,03^{\circ}$ aos 3 meses; e $19,62^{\circ}$; $8,14^{\circ}$ e $9,66^{\circ}$ aos 24 meses.

No grupo 2 (chevron), a correção média para o AMF, AIM e AAMD foi de $20,49^{\circ}, 6,47^{\circ} \mathrm{e} 10,63^{\circ}$ respectivamente aos 3 meses; e $19,47^{\circ}, 5,9^{\circ}$ e $9,5^{\circ}$ aos 24 meses. Os resultados relativos às correções angulares em ambos os grupos foram semelhantes (- Tabela 2 ).

\section{Medições pré-operatórias}

1. A distribuição da congruência pré-operatória por técnica pode ser vista na - Tabela 3. As diferenças na distribuição da congruência entre ambas as técnicas foram estatisticamente significativas (valor de $p$ : 0,008).

2. Foi observada diferença significativa somente no AIM préoperatório de ambos os grupos (valor de $p$ : 0,012). Os outros ângulos pré-operatórios em ambos os grupos não apresentaram diferenças significativas (-Tabela 4 ).

\section{Medição em 3 meses de pós-operatório e comparação com pré-operatório.}

1. Foram encontradas diferenças significativas entre a congruência do AMF pré-operatória e pós-operatória aos 3

Tabela 2 Correção média em graus dos ângulos em ambos os grupos em 3 e 24 meses

\begin{tabular}{|l|l|l|l|l|}
\hline $\begin{array}{l}\text { Correção } \\
\text { média }\end{array}$ & $\begin{array}{l}\text { Bosch } \\
\text { 3 meses }\end{array}$ & $\begin{array}{l}\text { Chevron } \\
\text { 3 meses }\end{array}$ & $\begin{array}{l}\text { Bosch } \\
\text { 24 meses }\end{array}$ & $\begin{array}{l}\text { Chevron } \\
\text { 24 meses }\end{array}$ \\
\hline AMF & 20,19 & 20,49 & 19,62 & 19,47 \\
\hline AIM & 8,66 & 6,47 & 8,14 & 5,9 \\
\hline AAMD & 9,03 & 10,63 & 9,66 & 9,5 \\
\hline
\end{tabular}

Abreviaturas: AAMD, ângulo articular metatarsodistal; AIM, ângulo intermetatarsal; AMP, ângulo metatarsofalângico.
Tabela 3 Distribuição da congruência conjunta pré-operatória em ambos os grupos

\begin{tabular}{|l|l|l|l|}
\hline & $\begin{array}{l}\text { Nenhuma } \\
\text { congruência }\end{array}$ & Congruência & Total \\
\hline Bosch & 20 & 42 & 62 \\
\hline Chevron & 8 & 55 & 63 \\
\hline Total & 28 & 97 & 125 \\
\hline
\end{tabular}

meses em pacientes tratados com a técnica de Bosch: Teste de McNemar valor de $p=0,00225$. Não há diferenças registradas na técnica chevron.

2. Quanto à correção angular, comparamos a evolução de cada ângulo por grupo (Bosch e chevron) entre o préoperatório e 3 meses e não encontramos diferenças estatisticamente significativas. No entanto, a TR para o AIM de 3 meses foi de $63 \%$ na técnica de Bosch, enquanto em pacientes tratados com chevron foi de $55 \%$ $(p=0,0668)$. Embora não estatisticamente significativa, essa diferença mostra uma maior correção potencial de AIM com técnica Bosch.

\section{Medição aos 24 meses após cirurgia e comparação com o período pré-operatório.}

1. Não houve diferenças estatisticamente significativas entre a congruência pré-operatória e o pós-operatório aos 24 meses em pacientes tratados com técnica em chevron: valor de $p=0,72$, mas encontramos diferenças significativas nos pacientes tratados com a técnica de Bosch: valor de $p=0,0013$

2. As evoluções por técnica foram comparadas em cada um dos ângulos considerados, entre as medições pré-operatórias e as medidas aos 24 meses, e foi encontrada diferença estatisticamente significativa no AIM entre as técnicas de Bosch e em chevron (- Tabela 5).

3. Não houve diferenças na evolução de ambos os grupos (3-24 meses) no AIM, AMP e AAMD ( $p>0,79)$. Portanto, esses ângulos mantiveram a correção em longo prazo.

Complicações: Não houve complicações intraoperatórias em nenhum dos grupos.

Houve 10 complicações pós-operatórias no grupo operado com a ténica de Bosch e 7 no grupo tratado com a técnica em chevron. Entretanto, a incidência de complicações entre as duas técnicas foi semelhante (teste do ${ }^{\circ}$ qui-quadrado, valor de $p=0,41)$. A partir da análise de complicações, no grupo 1 (Bosch) foram encontrados 5 casos de celulite na inserção de fio $\mathrm{K}, 1$ ruptura de implante, 1 neurite, 1 osteomielite, 1 hematoma e um caso de trombose venosa profunda. No grupo 2 (chevron), foram encontrados 3 casos de celulite, 2 de neurite, 1 reincidência e 1 hálux varo.

Tempo cirúrgico: o tempo cirúrgico foi medido a partir de quando o paciente entrou na sala de cirurgia até sair da sala de cirurgia. Média do grupo chevron: 107 minutos; grupo Bosch: 50 minutos (valor de $p<0,0001$ ). 
Tabela 4 Comparação de ambos os grupos. Valores médios dos ângulos e significância estatística

\begin{tabular}{|l|l|l|l|l|l|l|l|}
\hline & Grupo 1 & Grupo 2 & Média (1) & Média (2) & DP (1) & DP (2) & Valor de $P$ \\
\hline AMF & Bosch & Chevron & 29,94 & 29,52 & 6,69 & 7,51 & 0,7686 \\
\hline AIM & Bosch & Chevron & 12,71 & 11,65 & 3,15 & 2,63 & $\mathbf{0 , 0 1 2 0}$ \\
\hline AAMD & Bosch & Chevron & 16,94 & 18,08 & 4,75 & 7,98 & 0,9171 \\
\hline
\end{tabular}

Abreviaturas: AAMD, ângulo articular metatarsodistal; AIM, ângulo intermetatarsal; AMP, ângulo metatarsofalângico; DP, desvio padrão.

Tabela 5 Comparação da correção potencial em ambos os grupos entre 1 mês pré-operatório e 24 meses pós-operatório

\begin{tabular}{|l|l|l|l|l|l|l|l|l|l|}
\hline Variável & Grupo 1 & Grupo 2 & $\begin{array}{l}\text { Média } \\
(\mathbf{1})\end{array}$ & $\begin{array}{l}\text { Média } \\
(\mathbf{2})\end{array}$ & DP (1) & DP (2) & $\begin{array}{l}\text { Med } \\
(\mathbf{1})\end{array}$ & $\begin{array}{l}\text { Med } \\
(\mathbf{2})\end{array}$ & Valor de $\boldsymbol{p}$ \\
\hline TR AMF 24 m & Bosch $(\mathrm{n}=62)$ & Chevron $(\mathrm{n}=63)$ & 0,66 & 0,64 & 0,19 & 0,19 & 0,69 & 0,68 & 0,5651 \\
\hline TR AIM 24 m & Bosch $(\mathrm{n}=62)$ & Chevron $(\mathrm{n}=63)$ & 0,58 & 0,49 & 0,27 & 0,24 & 0,59 & 0,46 & $\mathbf{0 , 0 1 8 8}$ \\
\hline TR AAMD 24 m & Bosch $(\mathrm{n}=62)$ & Chevron $(\mathrm{n}=63)$ & 0,47 & 0,44 & 0,34 & 0,31 & 0,56 & 0,50 & 0,4693 \\
\hline
\end{tabular}

Abreviaturas: AAMD, ângulo articular metatarsodistal; AIM, ângulo intermetatarsal; AMP, ângulo metatarsofalângico; DP, desvio padrão; TR, taxa de recuperação.

\section{Discussão}

A cirurgia minimamente invasiva aplicada ao pé tornou-se popular na década de 1980 , e vem crescendo na última década com base na teoria de obter resultados melhores naqueles pacientes que não se recuperaram bem das cirurgias abertas tradicionais.

Portaluri, ${ }^{18}$ em 2000, e depois Magnan et al., ${ }^{20}$ em 2008, publicaram uma série de casos com 118 pés tratados com a técnica de Bosch sem parafuso, com um seguimento médio de 35,9 meses, e mencionaram que os resultados clínicos foram comparáveis aos obtidos através de cirurgia aberta convencional.

Em 2007, Migues et al. ${ }^{21}$ publicaram sua experiência avaliando uma série de 242 pés tratados com técnica de Bosch, baseando a medição em milímetros de deslocamento da cabeça.

Analisando a bibliografia internacional, encontramos muitas séries de casos de cirurgia minimamente invasiva, e pouquíssimas comparações dessa técnica com a convencional. ${ }^{22-24}$ A correção média relatada na cirurgia minimamente invasiva para AMFs e iAIMs é de $26,7^{\circ}-33,3^{\circ}$ e $11,8^{\circ}-13^{\circ}$, respectivamente. ${ }^{25-27}$

Os resultados obtidos no grupo 1, tratados de forma minimamente invasiva, hálux valgo e AIM de $29,66^{\circ}$ e $13,29^{\circ}$, respectivamente, são comparáveis aos publicados.

Giannini et al. ${ }^{22}$ realizaram estudo randomizado comparando os resultados clínico-radiológicos do tratamento de 40 pacientes com hálux valgo bilateral. Em um pé, eles fizeram uma osteotomia aberta de cachecol, e no outro pé, uma cirurgia Bosch percutânea tradicional sem parafuso. Não encontraram diferença estatisticamente significativa nas medidas angulares pós-operatórias. No entanto, a cirurgia percutânea produz tempos cirúrgicos menores. Concluíram que ambas as técnicas são eficazes na correção da deformidade, mas eles acreditam que o menor tempo cirúrgico, a menor agressividade e a simplicidade na estabilização da cirurgia percutânea melhoram os desfechos clínicos. De acordo com as publicações, em nosso estudo, obtivemos correções angulares semelhantes em ambos os grupos, mas o tempo cirúrgico na cirurgia percutânea de Bosch foi inferior.

Brogan ${ }^{28}$ compara osteotomia percutânea versus chevron convencional. Os escores clínicos e radiológicos pós-operatórios de todos os ângulos foram substancialmente melhorados em ambos os grupos $(p<0,001)$, mas não houve diferença estatisticamente significativa na melhora angular entre $o$ grupo tratado com cirurgia aberta e o grupo tratado com cirurgia minimamente invasiva $(p>0,05)$. Ele também não encontrou diferença significativa nas complicações $(p>0,5)$. Na sequência do autor, em nosso estudo, não houve diferenças estatisticamente significativas entre os grupos relativas a correções angulares e complicações (valor $p=0,41$ ).

Uma fraqueza do nosso estudo é que não correlacionamos os resultados radiológicos com os clínicos, embora o objetivo fosse fazer uma análise radiológica. Entretanto, realizou-se uma análise estatística completa comparando duas técnicas, que não foram analisadas em outros estudos, e avaliando correções angulares radiográficas ao longo do tempo (médio prazo), bem como possível perda de correção. Por essa razão, não concordamos que ambas as técnicas sejam semelhantes na correção de todos os ângulos, uma vez que encontramos uma potencial correção a médio prazo do AIM no grupo tratado com a técnica de Bosch. No entanto, ambas as técnicas apresentam comportamento semelhante na evolução ao longo do tempo e não apresentam diferenças estatisticamente significativas na perda de correção.

Ianno et al. ${ }^{19}$ publicaram uma série de casos de cirurgia percutânea, utilizando uma técnica de Bosch sem osteossíntese, e relataram alta incidência de complicações; entre eles, 4 casos de consolidação viciosa, osteonecrose e 16 casos de recidivas. Nosso estudo, com um número estatisticamente significativo de técnicas de Bosch, não apresentou recidivas, osteonecrose ou hálux varo.

\section{Conclusões}

Ambas estabilizadas com parafusos, tanto a cirurgia de Bosch quanto a osteotomia em chevron (híbrida quando associada à 
osteotomia percutânea de Akin) apresentam correção adequada de hálux valgo. No entanto, os pacientes tratados com cirurgia percutânea de Bosch apresentaram maior poder de correção do AIM em médio prazo e menor tempo cirúrgico quando comparados com os pacientes tratados com a osteotomia em chevron,. Ambas as técnicas se comportaram da mesma forma na evolução ao longo do tempo no que diz respeito à perda de correção e complicações pós-operatórias.

\section{Suporte Financeiro}

Não houve suporte financeiro de fontes públicas, comerciais, ou sem fins lucrativos.

Conflito de interesses

Os autores não têm conflito de interesses para declarar.

\section{Referências}

1 Mann RA, Coughlin MJ. Hallux valgus-etiology, anatomy, treatment and surgical considerations. Clin Orthop Relat Res 1981; (157):31-41

2 Coughlin MJ. Hallux valgus in men: effect of the distal metatarsal articular angle on hallux valgus correction. Foot Ankle Int 1997; 18(08):463-470

3 Coughlin MJ, Thompson FM. The high price of high-fashion footwear. Instr Course Lect 1995;44:371-377

4 Baravarian B, Briskin GB, Burns P. Lapidus bunionectomy: arthrodesis of the first metatarsocunieform joint. Clin Podiatr Med Surg 2004;21(01):97-111

5 Biz C, Corradin M, Petretta I, Aldegheri R. Endolog technique for correction of hallux valgus: a prospective study of 30 patients with 4-year follow-up. J Orthop Surg Res 2015;10:102

6 Austin DW, Leventen EO. A new osteotomy for hallux valgus: a horizontally directed "V" displacement osteotomy of the metatarsal head for hallux valgus and primus varus. Clin Orthop Relat Res 1981;(157):25-30

7 Schneider W, Aigner N, Pinggera O, Knahr K. Chevron osteotomy in hallux valgus. Ten-year results of 112 cases. J Bone Joint Surg Br 2004;86(07):1016-1020

8 Maffulli N, Longo UG, Marinozzi A, Denaro V. Hallux valgus: effectiveness and safety of minimally invasive surgery. A systematic review. Br Med Bull 2011;97:149-167

9 Bosch P, Markowski H, Rannicher V. Technik und erste ergebnisse der subkutanen distalen metatarsale-I osteotomie. Orthop Praxis 1990;26:51-56

10 De Prado M, Ripoll PL, Golano P. Minimally invasive foot surgery: surgical techniques, indications, anatomical basis. Barcelona: About Your Health Publishers; 2009

11 Perera AM, Redfern A, Singh D, Lomax A. Minimally invasive forefoot surgery. J Trauma Orthop 2015;3(01):50-55

12 Yáñez Arauz JM, Del Vecchio J, Eksarho A, Ghioldi M, Escobar G, Yáñez Arauz ME. Cirugía "hibrida" del hallux valgus moderado: resultado radiológicos a mediano plazo. Hybrid surgery for moderate hallux valgus: midterm radiographic results. Tobillo y Pie 2015;7(01):43-47

13 Coughlin MJ. Hallux valgus. J Bone Joint Surg Am 1996;78(06): 932-966

14 Chiang CC, Lin CF, Tzeng YH, Huang CK, Chen WM, Liu CL. Distal linear osteotomy compared to oblique diaphyseal osteotomy in moderate to severe hallux valgus. Foot Ankle Int 2012;33(06): 479-486

15 Miller JW. Distal first metatarsal displacement osteotomy. Its place in the schema of bunion surgery. J Bone Joint Surg Am 1974;56(05):923-931

16 Deenik AR, de Visser E, Louwerens JW, de Waal Malefijt M, Draijer FF, de Bie RA. Hallux valgus angle as main predictor for correction of hallux valgus. BMC Musculoskelet Disord 2008;9:70

17 Jones KJ, Feiwell LA, Freedman EL, Cracchiolo A 3rd. The effect of chevron osteotomy with lateral capsular release on the blood supply to the first metatarsal head. J Bone Joint Surg Am 1995;77 (02):197-204

18 Portaluri M. Hallux valgus correction by the method of Bosch: a clinical evaluation. Foot Ankle Clin 2000;5(03):499-511

19 Iannò B, Familiari F, De Gori M, Galasso O, Ranuccio F, Gasparini G. Midterm results and complications after minimally invasive distal metatarsal osteotomy for treatment of hallux valgus. Foot Ankle Int 2013;34(07):969-977

20 Magnan B, Samaila E, Viola G, Bartolozzi P. Minimally invasive retrocapital osteotomy of the first metatarsal in hallux valgus deformity. Oper Orthop Traumatol 2008;20(01):89-96

21 Migues A, Campaner G, Slullitel G, Sotelano P, Carrasco M, Solari G. Minimally invasive surgery in hallux valgus and digital deformities. Orthopedics 2007;30(07):523-526

22 Giannini S, Faldini C, Vannini F, et al. Surgical treatment of hallux valgus: a clinical prospective randomized study comparing linear distal metatarsal osteotomy with scarf osteotomy. Orthop Proc 2009;91-B(Suppl 1):162

23 Maffulli N, Longo UG, Oliva F, Denaro V, Coppola C. Bosch osteotomy and scarf osteotomy for hallux valgus correction. Orthop Clin North Am 2009;40(04):515-524

24 Radwan YA, Mansour AM. Percutaneous distal metatarsal osteotomy versus distal chevron osteotomy for correction of mild-tomoderate hallux valgus deformity. Arch Orthop Trauma Surg 2012;132(11):1539-1546

25 Bauer T, de Lavigne C, Biau D, De Prado M, Isham S, Laffenétre O. Percutaneous hallux valgus surgery: a prospective multicenter study of 189 cases. Orthop Clin North Am 2009;40(04): 505-514

26 Huang PJ, Lin YC, Fu YC, Yang YH, Cheng YM. Radiographic evaluation of minimally invasive distal metatarsal osteotomy for hallux valgus. Foot Ankle Int 2011;32(05):S503-S507

27 Magnan B, Bortolazzi R, Samaila E, Pezzè L, Rossi N, Bartolozzi P. Percutaneous distal metatarsal osteotomy for correction of hallux valgus. Surgical technique J Bone Joint Surg Am 2006;88(Suppl 1 Pt 1):135-148

28 Brogan K, Lindisfarne E, Akehurst H, Farook U, Shrier W, Palmer S. Minimally Invasive and Open Distal Chevron Osteotomy for Mild to Moderate Hallux Valgus. Foot Ankle Int 2016;37(11): 1197-1204 